Gut, 1984, 25, 1065-1070

Alimentary tract and pancreas

\title{
Endotoxaemia and acute pancreatitis: correlation between the severity of the disease and the anti- enterobacterial common antigen antibody titre
}

\author{
E KIVILAAKSO, V V VALTONEN, M MALKAMÄKI, A PALMU, \\ T SCHRÖDER, P NIKKI, P H MÄKELÄ, AND M LEMPINEN
}

From the Second Department of Surgery, and Second Department of Medicine, and Intensive Care Unit, Helsinki University Central Hospital, and Department of Bacteriology and Immunology, University of Helsinki, and National Public Health Institute, Helsinki, Finland

SUMMARY Enterobacterial common antigen is a highly immunogenic component of the Gram negative bacterial cell wall that is common to all enteric bacteria. In the present study, the humoral antibody response against enteric bacteria was investigated by measuring antibodies to enterobacterial common antigen in paired serum samples in 38 patients with acute pancreatitis and in 31 healthy subjects. In mild pancreatitis (11 patients), no changes in anti-enterobacterial common antigen titres were observed as compared with healthy controls. Nine of the 10 patients had a significant increase ( $\geqslant 8$ times) in anti-enterobacterial common antigen titres during the disease. Similarly, in patients with fulminant (haemorrhagic) pancreatitis who survived, a significant increase in anti-enterobacterial common antigen titres occurred during the course of the disease (in nine of the 11 patients). Paradoxically, only one of the six patients with fulminant pancreatitis with fatal outcome showed a significant increase in his anti-enterobacterial common antigen titre. The results suggest that Gram negative bacterial components escape into the systemic circulation in acute pancreatitis. This may have pathophysiologic significance in this disease.

Caridis et $a^{1}$ and Fossar and Kakkar ${ }^{2}$ were first to describe endotoxaemia in patients with acute pancreatitis. This has lead to speculation that endotoxaemia might be of pathophysiologic significance in this disease, especially in the development of the various complications inherent to severe pancreatitis, such as shock, pulmonary insufficiency, disseminated intravascular coagulopathy and stress ulceration. $^{3}$

The biologically active component of endotoxin molecule, the endotoxic lipopolysaccharide, is difficult to measure quantitatively from body fluids. So far, the most sensitive assay for endotoxin has been the limulus lysate test. It is not, however, entirely specific for endotoxin, particularly if applied to serum or body fluids. ${ }^{4}$

Enterobacterial common antigen is an immunogenic component of the Gram negative bacterial cell

Address for correspondence: Eero Kivilaakso. MD. Second Department of Surgery, Helsinki University Central Hospital, 00290 Helsinki 29, Finland. Received for publication 4 January 1984 wall that is common to all enteric bacteria (family Enterobacteriaceae). Enterobacterial common antigen is spatially closely linked to the lipopolysaccharide - both being components of the outer membrane of the enteric bacteria. In some strains the enterobacterial common antigen immunodeterminant of Enterobacteriaceae is part of the lipopolysaccharide molecule itself. ${ }^{5}$ Because of the great serological heterogeneity of lipopolysaccharide of enteric bacteria, anti-enterobacterial common antigen determination is so far the only generally applicable assay for the humoral immune response to enteric bacteria (and indirectly, to endotoxin).

Raised anti-enterobacterial common antigen titres have been found in patients with alcoholic cirrhosis supporting the concept that enteric bacteria - possibly endotoxin - have a role in the pathophysiology of alcoholic cirrhosis. ${ }^{6}$

The present study investigates whether there is a humoral immune response to enteric bacteria in acute pancreatitis by assaying anti-enterobacterial 
common antigen antibody titres in such patients. Special emphasis was laid on the relationship of anti-enterobacterial common antigen titres with the severity of the disease as well as with the presence of manifest Gram negative infection.

\section{Methods}

\section{PATIENTS}

Thirty eight patients treated for acute pancreatitis at the Second Department of Surgery, Meilahti Hospital, in 1979-1981 were included in the series. The diagnosis was established clinically all patients having raised serum amylase $(>5000$ Phadebas units). The severity of the disease was assessed, in part, using the prognostic criteria as advocated by Ranson et al. ${ }^{7}$ According to this scale, the presence of three or more of the 11 prognostic signs denotes to severe pancreatitis with deteriorated prognosis. The patients were divided in four groups as follows:

\section{Group A: mild pancreatitis}

Eleven patients (three women, eight men; mean age 44 years). In this group were included patients whose hospital stay was less than 10 days and who had no complications. Average number of prognostic signs was 0 and average hospital stay eight days. The aetiologic cause was alcohol in 10 patients and unknown in one patient.

\section{Group B: moderate pancreatitis}

Ten patients (three women, seven men; mean age 43 years). In this group were included patients whose hospital stay was more than 14 days with or without complications. Average number of prognostic signs was 1.3 and the average hospital stay 20 days. The aetiologic cause was alcohol in seven patients, biliary tract disease in one patient and unknown in two patients. Three of the 10-patients developed a pseudocyst as a complication, whereas seven patients had only prolonged course of the disease without specific complications.

\section{Group C: fulminant pancreatitis with survival}

Eleven patients (two women, nine men; mean age 37 years). In this group were included those patients with fulminant (haemorrhagic) pancreatitis verified at laparotomy, who survived their disease. The average number of prognostic signs was 3.6 and average hospital stay 42 days. The aetiologic cause was alcohol in nine patients, biliary tract disease in one patient and unknown in one patient.

Group D: fulminant pancreatitis with fatal outcome Six patients (six men; mean age 46 years). This group includes patients suffering from fulminant (haemorrhagic) pancreatitis verified at laparotomy, who ultimately died of their disease. The average number of prognostic signs was $5 \cdot 3$ and average hospital stay was 39 days. The aetiologic cause was alcohol in five patients and unknown in one patient.

\section{CONTROLS}

In addition, 31 healthy subjects (23 women, eight men; mean age 33 years) with no recent history of infectious diseases served as controls.

\section{MANAGEMENT OF THE PATIENTS}

Mild pancreatitis was treated by nasogastric suction and intravenous fluids. Moderate pancreatitis was managed in a similar manner but in addition antibiotics (ampicillin, cephalosporin) were administered if prolonged fever or pseudocyst was present. When fulminant pancreatitis was suspected clinically, a laparotomy was performed and the diagnosis was verified, whereafter the patient was treated at random either by subtotal pancreatic resection (see ref. 8) or peritoneal lavage via cannulas inserted at the operation in the peripancreatic area $(1000 \mathrm{ml} / \mathrm{h}$ peritoneal dialysis solution for five to seven days). The routine postoperative care in both methods of treatment included: intravenous fluids with total parenteral nutrition until oral intake of food was resumed, ventilatory support, prophylactic antibiotics (tobramycin + clindamycin) and stress ulcer prophylaxis (cimetidine and oral antacids in doses sufficient to maintain intragastric $\mathrm{pH}$ continuously above $5 \cdot 0$ ).

\section{ENTEROBACTERIAL COMMON ANTIGEN ANTIBODY} ASSAY

Enterobacterial common antigen antibody was measured with passive haemagglutination method as described in detail earlier. ${ }^{9}$ The titre 1:4096 corresponds to the 98th percentile limit in healthy Finnish adult population. The serum samples from the patients were drawn on admission and weekly thereafter for at least three weeks. One serum sample was drawn from the healthy controls. The sera were kept at $-20^{\circ} \mathrm{C}$ until analysed.

\section{STATISTICAL METHODS}

For statistical analysis Student's $t$ test and $\chi^{2}$ test with Yates correction for small numbers were used.

\section{Results}

CORRELATION BETWEEN SEVERITY OF THE

DISEASE AND THE ENTEROBACTERIAL COMMON ANTIGEN TITRES

Group $A$ 
Table 1 Enterobacterial common antigen antibody titres in patients with acute pancreatitis

\begin{tabular}{llccc}
\hline & & & & $\begin{array}{l}\text { Incidence of } \\
\text { significant titre } \\
\text { increase ( } \geqslant 8 \text { times) }\end{array}$ \\
\hline Severity of disease & Patients (no) & Admission & Maximal & $0 / 11$ \\
Moderate/complicated (B) & 11 & $389 \cdot 9 *(64-2048)$ & $545(64-2048)$ & $9 / 10$ \\
Fulminant survival (C) & 10 & $97 \cdot 0(32-512)$ & $1176 \cdot 3(512-2048)$ & $9 / 11$ \\
Fulminant death (D) & 11 & $226 \cdot 0(32-1024)$ & $3396(64-65536)$ & $1 / 6$ \\
Controls & 6 & $50 \cdot 9(16-128)$ & $90 \cdot 5(16-256)$ & - \\
\hline
\end{tabular}

* Geometric mean (ranges)

The anti-enterobacterial common antigen titres of patients with mild, oedematous pancreatitis did not differ on admission significantly from those of healthy controls (Table 1). Also, none of the 11 patients subsequently experienced any significant increase $(\times 8$ or more) in their anti-enterobacterial common antigen titres.

\section{Group B}

Patients with moderate pancreatitis (prolonged and/ or complicated course of the disease) had significantly $(\mathrm{p}<0.01)$ decreased anti-enterobacterial common antigen titres on admission as compared with the controls (Table 1 ). Nine of the 10 patients had a significant increase in anti-enterobacterial common antigen titre during the course of the disease.

\section{Group C}

Patients surviving after haemorrhagic pancreatitis also had significantly $(p<0.05)$ decreased antienterobacterial common antigen titres on admission as compared with controls (Table 1). During the course of the illness, a marked increase in antienterobacterial common antigen titres occurred. This was much more extensive than in patients of group B. Individually, in nine of the 11 patients a significant increase in anti-enterobacterial common antigen titres occurred. Six of these patients were treated by peritoneal lavage and five by pancreatic resection. The maximal anti-enterobacterial common antigen titres were somewhat, but not significantly, higher among patients with peritoneal lavage (4096 vs 2702).

\section{Group D}

In six patients, who succumbed to haemorrhagic pancreatitis and had the most severe form of the disease (average score of positive prognostic signs 5.53 as compared with 3.55 in group $C$ ), the anti-enterobacterial common antigen titre behaved in a completely different way. On admission the average anti-enterobacterial common antigen titre was significantly $(\mathrm{p}<0.01)$ lower than in the controls but in only one of the six patients a significant increase in anti-enterobacterial common antigen titre subsequently occurred during the average 38 days (range 12-74 days) hospital stay (Table 1). Three patients were treated by peritoneal lavage and three patients with pancreatic resection. Again, the maximal anti-enterobacterial common antigen titres were somewhat, but not significantly, higher among patients with peritoneal lavage (161 vs 51).

\section{SEPTICAEMIA}

No septic infections were recorded in patients of groups $\mathrm{A}$ and $\mathrm{B}$.

Three patients in group $\mathrm{C}$ had positive blood cultures during hospital stay. In one of them, a Gram negative rod (Enterobacter cloacae) was identified as the causative agent, and she experienced a significant increase in her antienterobacterial common antigen titre (1024 to 8192). One patient had Candida albicans septicaemia but likewise experienced a significant increase in anti-enterobacterial common antigen titre (512 to 16384 ), whereas the third patient with Staphyloccus epidermidis as the causative agent experienced no increase in his anti-enterobacterial common antigen titre.

Four patients in group $\mathrm{D}$ had positive blood cultures. Two of them had Gram negative bacteraemia caused by $E$. coli and Proteus mirabilis. None of these patients had any increase in their anti-enterobacterial common antigen titre.

\section{ABSCESS}

Four patients in group $\mathrm{C}$ had an intra-abdominal abscess verified (and drained) at laparotomy. In two of them the abscess was caused by Gram negative enterobacteria. One patient, having an $E$ coli abscess, had a significant increase in her antienterobacterial common antigen titre (512 to 16384 ), whereas the other, having Enterobacter cloacae as the causative agent, had no increase in his anti-enterobacterial common antigen titre. The 
remaining two patients had Staphylococcus epidermidis as the causative agent and both of them experienced a very strong increase in their antienterobacterial common antigen titres (256 to $65536)$.

Two patients in group $D$ had intra-abdominal abscesses. In both cases, the causative agent was $E$ coli, but neither of the patients had a significant increase in their anti-enterobacterial common antigen titres.

\section{OTHER INFECTIONS}

Six patients in group $\mathrm{C}$ had positive cultures from intra-abdominal drains and/or trachea or had wound infection. In no instance, however, was the causative agent a Gram negative bacterium (Staphyloccus epidermidis in five patients, Staphylococcus aureus in one, Candida albicans in one).

Four patients in group D had positive drain or tracheal cultures. In three cases Gram negative bacteria were among the causative agents $(E$ coli and Pseudomonas in each). All these patients had also $E$ coli sepsis and/or abscesses. None of the patients experienced an increase in their antienterobacterial common antigen titres.

In Table 2 is shown the relationship between verified infection (sepsis, abscess, positive cultures from intra-abdominal drains or trachea, or wound infection) and anti-enterobacterial common antigen titres in group C. No correlation was observed, no matter whether or not the infection was caused by Gram negative bacteria.

\section{Discussion}

The present results show that in the mildest form of acute pancreatitis (Group A), no significant changes

Table 2 Relationship between the manifest infection and enterobacterial common antigen antibody titre in patients with fulminant pancreatitis (Group C)

\begin{tabular}{llll}
\hline $\begin{array}{l}\text { Type of } \\
\text { infection }\end{array}$ & $\begin{array}{l}\text { Patients } \\
\text { (no) }\end{array}$ & $\begin{array}{l}\text { Maximal titre } \\
\text { (geometric } \\
\text { mean) }\end{array}$ & $\begin{array}{l}\text { Incidence of } \\
\text { significant } \\
\text { titre increase } \\
(\geqslant 8 \text { times })\end{array}$ \\
\hline $\begin{array}{l}\text { Sepsis } \\
\quad \text { Gram negative }\end{array}$ & 1 & 8192 & $1 / 1$ \\
$\begin{array}{l}\text { Other } \\
\text { Abscess }\end{array}$ & 2 & 1024 & $1 / 2$ \\
$\quad \begin{array}{l}\text { Gram negative } \\
\text { Other }\end{array}$ & 2 & 1024 & $1 / 2$ \\
$\begin{array}{l}\text { Other infection } \\
\text { Gram negative }\end{array}$ & 2 & 65536 & $2 / 2$ \\
$\quad$ Other & 6 & - & $-1 / 6$ \\
No infection & 5 & 6517 & $4 / 5$ \\
\hline
\end{tabular}

in anti-enterobacterial common antigen titres occurred as compared with the controls. The antienterobacterial common antigen titres were normal on admission and remained unchanged during the subsequent course of the disease, thus suggesting absence of significant infection or endotoxaemia in these patients.

In contrast, patients with more severe forms of the disease did experience profound alterations in their anti-enterobacterial common antigen titres. Interestingly, patients with moderate and severe pancreatitis (Group B, C, and D) had significantly decreased anti-enterobacterial common antigen titres on admission as compared with the controls. Although the reason and exact mechanism of this decrease in anti-enterobacterial common antigen titre remains to be delineated, one can speculate that it might be related to escape of enterobacterial common antigen and endotoxin from portal blood (where lipopolysaccharide is regularly encountered ${ }^{10}$ into the systemic circulation owing to impaired hepatic reticuloendothelial system function at the very beginning of pancreatitis. Hepatic reticuloendothelial clearance function is known to be depressed, for example, by ethanol ${ }^{11}$ and excessive ingestion of ethanol is by far the most common aetiologic factor of pancreatitis in these patients. This would lead to consumption of circulating anti-enterobacterial common antigen antibodies because of their conversion to immune complexes. A similar drop in anti-enterobacterial common antigen titres, speculatively explicable in terms of the same mechanism, has also been observed to precede the onset of manifest sepsis in some patients with severe haematological disorders, such as leukaemia (our unpublished observations).

Subsequently, a significant increase in antienterobacterial common antigen titres occurred in most of these patients (Groups B and C) during the course of the disease. The magnitude of this increase was dependent on the severity of the disease and in general the values measured in patients with haemorrhagic pancreatitis (with survival; Group C) were higher than we have ever measured for any disease entity so far. Yet no relationship was observed between the absolute value or relative increase of the anti-enterobacterial common antigen titres and the presence of Gram negative sepsis or other infection.

Curiously, no increase in anti-enterobacterial common antigen titre was observed in patients with the most fulminant form of the disease (patients with fatal outcome, who had also the highest score of poor prognostic signs; Group D). Those patients probably had the largest amount of endotoxaemia and enterobacterial common antigen in their blood; 
yet only one of the six patients had a significant increase in enterobacterial common antigen titre, and even his titres were relatively low. The time interval between the paired sera was about the same in each group and therefore does not explain the observed differences between the groups.

We have no good explanation for this paradoxical behaviour of the anti-enterobacterial common antigen antibodies. One possibility is some kind of immunogenic paralysis caused by overwhelming endotoxaemia in group D. ${ }^{12}$ Another possibility might be extensive consumption of the produced anti-enterobacterial common antigen antibodies to immune complex. Without turnover studies of the anti-enterobacterial common antigen antibodies it is difficult to decide between these two explanations, but the low level of anti-enterobacterial common antigen antibodies on admission in patients with pancreatitis favours the latter one. Limulus assays were not done on the same group of patients, because they are technically difficult to do when using serum samples and are not a very reliable indicator of endotoxaemia. ${ }^{413}$

Several clinical manifestations and complications frequently encountered in association with fulminant pancreatitis may be ascribed to excessive endotoxaemia. Fever, leukocytosis, hypotension, and shock are inherent clinical manifestations of fulminant pancreatitis and also signs of endotoxaemia. ${ }^{14-17}$ Adult respiratory distress syndrome also has been associated with fulminant pancreatitis, and endotoxaemia and certain complement components have been thought to play a role in the pathogenesis of adult respiratory distress syndrome. ${ }^{18}{ }^{19}$ In the stomach, endotoxin has been shown to promote acute gastric mucosal lesions. ${ }^{20}$ Other complications of fulminant pancreatitis that, theoretically, might be attributable to endotoxaemia include coagulopathy (DIC), ${ }^{21}{ }^{22}$ jaundice, ${ }^{23}$ renal failure, ${ }^{2425}$ and cardiac failure. ${ }^{26}$

The association of systemic endotoxaemia with acute pancreatitis, in particular severe and complicated forms of the disease, has been emphasised also in two other recent studies. Using the limulus lysate assay, Liehr et $a^{27}$ found endotoxaemia in $66 \%$ of patients with acute pancreatitis, and the presence of endotoxaemia seemed to correlate with the outcome and severity of the disease, as nine of their 21 patients with endotoxaemia died, whereas all 11 patients without endotoxaemia survived. Also systemic complications, in particular pulmonary and renal insufficiency, were more frequent among the patients with endotoxaemia. Similarly, Foulis $e a^{28}$ reported endotoxaemia in $50 \%$ of patients with acute pancreatitis and, again, mortality and incidence of extrapancreatic organ complications were higher among the patients who had endotoxaemia. Although these studies, together with the present findings, suggest a close relationship between endotoxaemia and severe and complicated forms of acute pancreatitis, it remains, of course, still unsettled whether the fatal outcome or complicated course of the illness are direct consequences of circulating endotoxin or whether endotoxaemia only coexist with other manifestations and complications of acute pancreatitis in the most severely ill patients. A low anti-enterobacterial common antigen titre in successive serum samples in patients with haemorrhagic pancreatitis might be an early indication of poor prognosis. Correspondingly, a significant increase in the anti-enterobacterial common antigen titre would indicate a favourable prognosis in patients with moderate or severe pancreatitis.

This work is supported in part by the Sigrid Jusélius Foundation, Helsinki, Finland.

\section{References}

1 Caridis DT, Reinhold RB, Woodruff PWH, Fine J. Endotoxemia in man. Lancet 1972; 2: 1381-6.

2 Fossard DP, Kakkar VV. The limulus test in experimental and clinical endotoxemia. Br J Surg 1974; 61: 798-804.

3 Fine J. Acute pancreatitis. Lancet 1975; 1: 1092.

4 Strumacher RJ, Kovnat MJ, McCabe WR. Limitation of the usefulness of the limulus assay for endotoxin. $N$ Engl J Med 1973; 288: 1261-4.

5 Mäkelä $\mathrm{PH}$, Mayer $\mathrm{H}$. Enterobacterial common antigen. Bacteriol Rev 1976; 40: 591-632.

6 Turunen U, Malkamäki M, Valtonen VV et al. Endotoxin and liver diseases. Gut 1981; 22: 849-53.

7 Ranson JHC, Rifkind KM, Roses DF, Fink SD, Eng $\mathrm{K}$, Spencer FC. Prognostic signs and the role of operative management in acute pancreatitis. Surg Gynecol Obstet 1974; 139: 69-81.

8 Kivilaakso E, Fräki O, Nikki P, Lempinen M. Resection of the pancreas for acute fulminant pancreatitis. Surg Gynecol Obstet 1981; 152: 493-8.

9 Malkamäki M. Antibodies to the enterobacterial common antigen: standardization of the passive hemagglutination test and levels in normal human sera. J Clin Microbiol 1981; 13: 1074-9.

10 Jacob AI, Goldberg PK, Bloom N, Degensheim GA, Kozinn PJ. Endotoxin and bacteria in portal blood. Gastroenterology 1977; 72: 1268-70.

11 Vilayat M, Nolan JP. Alcohol induced depression of reticuloendothelial function in the rat. $J$ Lab Clin Med 1967; 70: 295-301.

12 Morrison DC, Ryan JL. Bacterial endotoxin and host immune response. Advances in Immunology 1979; 28: 293-450.

13 Elin RJ, Robinson RA, Levine et al. Lack of clinical 
usefulness of the limulus test in the diagnosis of endotoxemia. $N$ Engl J Med 1975; 293: 521-4.

14 Lillehei RC, Longerbeam JK, Bloch JH, Manax WS. Hemodynamic changes in endotoxin shock. In: Mills LJ, Moyer JH, eds. Shock and hypotension: pathogenesis and treatment. New York: Grune \& Stratton, 1965: 442-62.

15 Wood WB Jr. Studies on the cause of fever. $N$ Engl J Med 1958; 258: 1023-31.

16 Boggs DR, Chervenick PA, Marsch JC, Cartwright GE, Wintrobe MM. Neutrophil releasing activity in plasma of dogs injected with endotoxin. J Lab Clin Med 1968; 72: 177-91.

17 Christy JH. Pathophysiology of gram-negative shock. Am Heart J 1971; 81: 694-701.

18 Riede V, Hassenstein W, Costabel U. Pathologischanatomische Untersuchungen bei der respiratorischen Insuffizienz durch Schoc. III. Histomorphometrische Untersuchungen der Methylprednisolon-Wirkung auf die durch Endotoxin induzierte Schocklunge. Intensivmed 1978; 15: 119-23.

19 Hammerschmidt DE, Weaver LJ, Hudson LD, Graddock PR, Jacob HS. Association of complement activation and elevated plasma-C5a with adult respiratory distress syndrome. Lancet 1980; 1: 947-9.

20 Cheung LY, Stephenson LW, Moody FG, Torma MJ, Zalewsky C. Direct effects of endotoxin on canine mucosal permeability and morphology. J Surg Res 1975; 18: 417-25.

21 Garner R, Evensen SA. Endotoxin-induced intravascular coagulation and shock in dogs: The role of factor VII. Br J Haematol 1974; 27: 655-68.

22 Morrison DC, Cochrane CG. Direct evidence for Hageman factor (factor XII) activation by bacterial lipopolysaccharides (endotoxins). J Exp Med 1974; 140: 797-811.

23 Utili R, Abernathy CO, Zimmerman HJ. Cholestatic effects of Escherichia coli endotoxin on the isolated perfused rat liver. Gastroenterology 1976; 70: 248-53.

24 Hinshaw LB, Bradley GM, Carlson $\mathrm{CH}$. Effect of endotoxin on renal function in the dog. Am J Physiol 1959; 196: 1127-31.

25 Wilkinson SP, Gazzard BG, Arroyo V. Relation of renal impairment and haemorrhagic diathesis to endotoxaemia in fulminant hepatic failure. Lancet 1974; 1: 521-4.

26 Cho YW. Direct cardiac action of E. coli endotoxin. Proc Soc Exp Biol Med 1972; 14: 705-7.

27 Liehr H, Grün M, Seeling R, Seeling H-P. Endotoxinämie bei akuter Pankreatitis. Leber Magen Darm 1980; 10: 259-64.

28 Foulis AK, Murray WR, Galloway D et al. Endotoxemia and complement activation in acute pancreatitis in man. Gut 1982; 23: 656-61. 